\title{
The Cytology of Human Pacinian Corpuscles: Evidence for Sprouting of the Central Axon
}

\author{
Chizuka IDE, Tohru NitATORI ${ }^{1}$ and Bryce L. MUNGER ${ }^{2}$ \\ Department of Anatomy (Prof. C. IDE) ${ }^{1}$, Iwate Medical University School of Medicine, Morioka, Japan \\ and Department of Anatomy (Prof. B. L. Munger), ${ }^{2}$ The Milton S. Hershey Medical Center, The \\ Pennsylvania State University, Hershey, Pennsylvania, U. S. A.
}

Received May 15, 1987

Summary. During the course of the studies on non-traumatized Pacinian corpuscles from normal human adults, we have frequently encountered corpuscles which have an 'apparently multiple' innervation in both light and electron microscopic preparations. On closer inspection of serial sections for both light and electron microscopy, these 'apparently multiple' axon terminals have been found in fact to be branches of the main central axon within the inner core of the corpuscle. Sprouting occurred at the trunk or at the extreme tip of the main axon, and such sprouts extended in various directions from the central axon throughout the inner core, producing tortuous and complex patterns of this 'multiple' innervation. These axonal sprouts do not have separate inner cores separated from one another, but rather are embedded in a common inner core. The presence of a common inner core thus differentiates normal axonal sprouts from the experimentally or pathologically produced multiple innervation that results form regeneration of axons in a previously denervated corpuscle. We conclude that the inner core of Pacinian corpuscles is a unique micro-environment promoting sprouting of sensory axon in the normal human adult as well as juvenile Pacinian corpuscles.

Pacinian corpuscles are exquisitely sensitive mechanoreceptors typically found deep in palmar and plantar skin and also in large numbers in the cat mesentery. The present authors were in the course of re-evaluating the cytology of cat Pacinian corpuscles (MUNGER et al., 1987; IDE et al., 1987), when we learned that the each of us had human Pacinian corpuscles demonstrating branching of the central axon implying sprouting of the axon in the inner core of the corpuscle. We each attributed these findings to nerve degeneration and subsequent regeneration since our materials were from autopsies which obviously had preexisting disease and medical treatment. Our tentative conclusion was due in part to the fact that ZELENA $(1984 \mathrm{a}, \mathrm{b})$ has described branching and multiple innervation in Pacinian corpuscles of the rat foot following nerve transection. However, on closer inspection these human specimens were indeed normal and our cases were from young adults and one case a child where the possibility of nerve trauma was extremely low. The present report is thus a light and electron microscopic study of normal human Pacinian corpuscles and documents sprouting of the central axon within the inner core. We conclude that the micro-environment of the inner core of human Pacinian corpuscles is thus unique promoting sprouting of sensory axons. 


\section{MATERIALS AND METHODS}

The serial sets of paraffin sections were prepared in one laboratory (BLM) and the electron microscopy in another laboratory (CI). The two young male subjects aged 14 and 16 were autopsied shortly after death ( $1 \mathrm{hr}$ and $21 / 2 \mathrm{hrs})$ and perfused through the vascular system with $3 \%$ glutaraldehyde in $0.1 \mathrm{M}$ phosphate buffer. Tissue samples were taken for light and electron microscopy, but only the light microscopy material was adequate for cytologic purposes. Blocks of digital skin were fixed in $10 \%$ neutral buffered formalin and processed in paraffin. Sets of serial sections were stained with the silver impregnation of SEVIER and MUNGER (1965). Tissue samples were obtained from two surgically removed digits which were fixed by immersion in $10 \%$ neutral buffered formalin. One specimen was from a two-year-old female subject with a supernumerary thumb that was electively removed for cosmetic reasons. The second surgical specimen was a traumatic partial amputation of the thumb of a 28-year-old female surgically removed due to the extensive tissue damage proximally. The distal phalanx was spared from trauma. Both surgical specimens were fixed by immersion in $10 \%$ neutral buffered formalin processed in paraffin for serial sections and impregnated with silver as described above. Hundred micrometer-thick frozen sections of the surgical specimens were also stained with Winkelmann silver technique (WINKEL. MANN, 1960). In every case individual Pacinian corpuscles were traced and photographed with Kodak Technical Pan film and developed with POTA developer (Kodak Technical Bulletin P-255) to enhance contrast of blackened axons (RICE and MUNGER, 1986).

The specimens prepared for electron microscopic observations were obtained from normal males aged 39 and 20. The volar skin of the fourth finger's distal phalanx was surgically opened and a small amount of subcutaneous tissues was excised. In addition we used Pacinian corpuscles obtained from toes of the amputated leg of a 19-year-old male following a traffic accident. Specimens were immersed immediately in fixative containing 2.5\% paraformaldehyde and $2.5 \%$ glutaraldehyde in $0.1 \mathrm{M}$ cacodylate buffer ( $\mathrm{pH}$ 7.4) and examined under a dissecting microscope for the presence of Pacinian corpuscles.

Pacinian corpuscles were stored in the fixative for one day and post-fixed at $4{ }^{\circ} \mathrm{C}$ in $1 \%$ osmium tetroxide solution for $2 \mathrm{hrs}$. The specimens were then processed in a conventional manner for electron microscopic observations. Serial thick $(1 \mu \mathrm{m})$ and thin sections were cut transversely to the long axis of the corpuscle in a way that two or three thin sections were harvested after cutting every five thick sections.

\section{RESULTS}

\section{Light microscopy}

By light microscopy several Pacinian corpuscles could be traced serially for many sections. Many corpuscles have only one central axon as noted in Figure 1. In these examples we could find no sign of branching over many serial sections. The inner core is homogeneous in appearance (Fig. 1b, c and see also Fig. 2-4). The majority of human Pacinian corpuscles resembles those depicted in Figure 1.

While we did not attempt to quantitiate the results, at least a quarter of human 


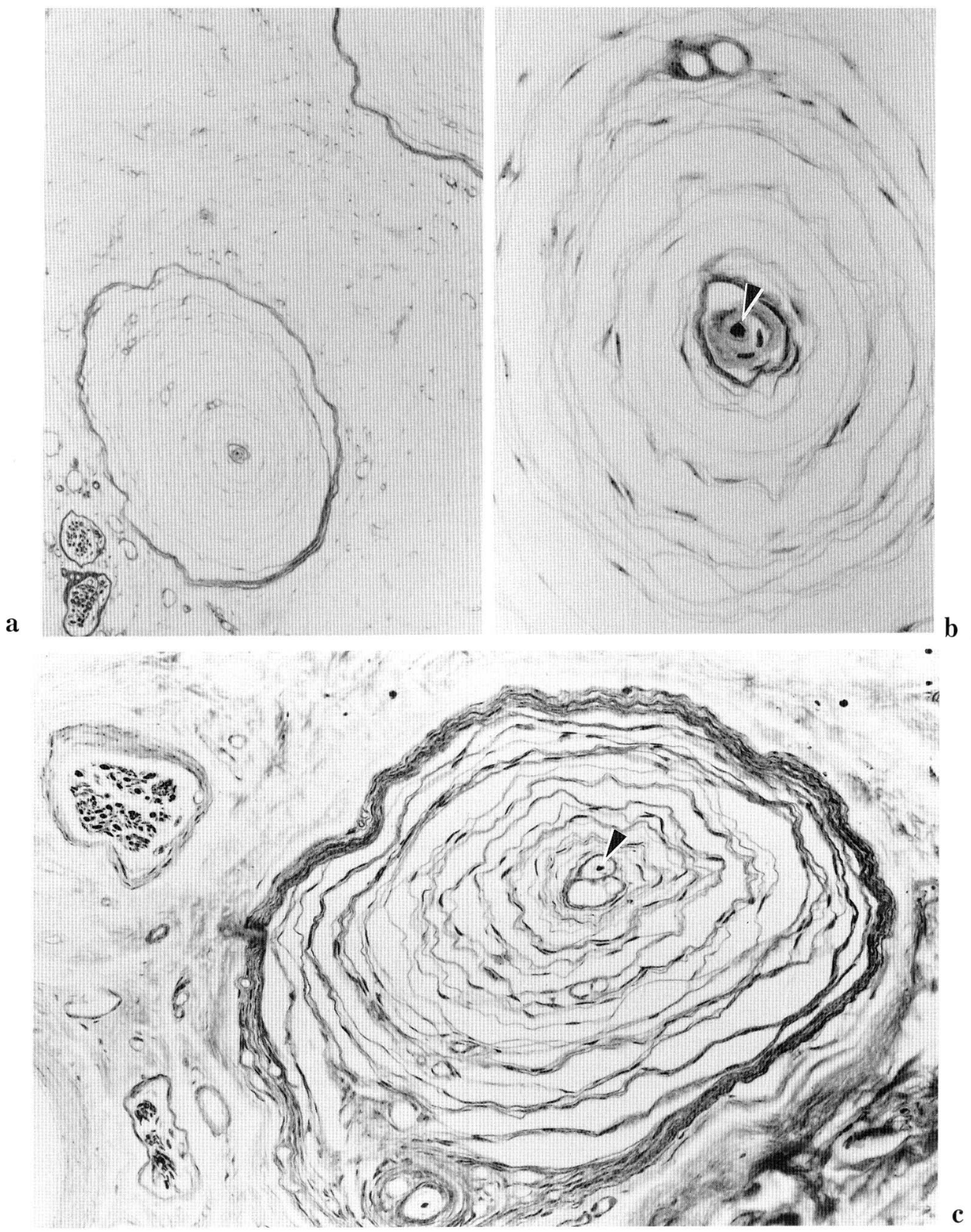

Fig. 1. a-c. These light micrographs have been taken from autopsied two normal human Pacinian corpuscles and both only have one central axon. a is a survey magnification and part of it is illustrated as a higher magnification in $\mathbf{b}$. $\mathbf{c}$ has been photographed at intermediate magnification to illustrate the entire extent of the outer core. a and b are almost a perfect cross section as indicated by the serial sections from which these photographs are taken. The central axon in a can barely visualized but can be seen clearly to be only one axon in $\mathbf{b}$. The other profiles in $\mathbf{b}$ to the right of the axon (arrowhead) are nuclei of inner core cells. The numerous capillaries in the outer core can be seen in $\mathbf{b}$ and $\mathbf{c}$. At least 20 outer lamellae can be counted in $\mathbf{c}$. a: $\times 80, \mathrm{~b}$ : $\times 400, c: \times 200$ 


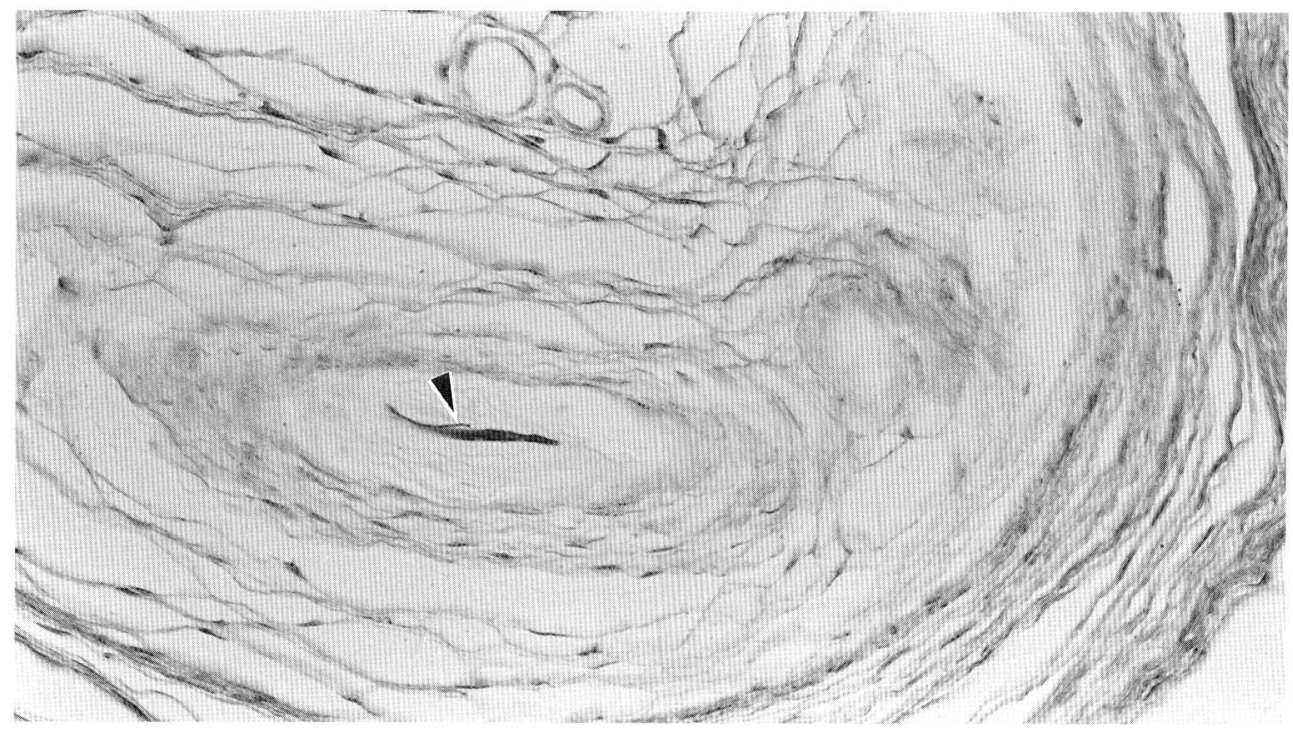

Fig. 2. The inner core has been sectioned longitudinally in this Pacinian corpuscle and a branching of the central axon can be seen at the arrowhead. The inner core is homogeneous in appearance and extends from the central axon to the base of the arrowhead where the first lamellae of the outer core can be seen totally encircling the inner core. $\times 400$

Pacinian corpuscles demonstrate branching of the central axon (Fig. 2-4). The course of the branches is complex. Some branches course proximally towards the myelinated axon and other distally towards the end of the corpuscle (Fig. 3). The branches can be extremely fine (Fig. 2) and in other cases large measuring more than $10 \mu \mathrm{m}$ in diameter (Fig. 3, 4). The axoplasm in the silver preparations frequently demonstrates fine internal structure suggesting non-uniformity of the substrate for silver deposition (Fig. $3)$. The inner core around the branches is homogeneous and uniform in texture. This important point can be used to distinguish reinnervated Pacinian corpuscles from normal Pacinian corpuscles. As described by ZELENÁ (1984b) and confirmed in unpublished studies of the authors on human digital skin following reinnervation, the axons in reinnervated corpuscles are only surrounded by a thin investment of lamellar cell cytoplasm, and we will return to this point in the Discussion.

\section{Electron microscopy - single axon}

By electron microscopy the Pacinian corpuscles have two distinct regions, an inner core (Fig. 5, 6) surrounding the central axon and an outer core (Fig. 7-9) of complete concentric lamellae separated by wide connective tissue compartments. (Note: the Figures are grouped with those housing a single axon in Figures 5 and 6 and those with multiple axons in Figures 7-9). The entire corpuscle is surrounded by a capsule that is continuous with the perineurium of the innervating nerve (SHANTHAVEERAPPA and Bourne, 1963). The capsule of human Pacinian corpuscles resembles the capsule of other corpuscular receptors (MUnGER, 1971; HALATA, 1975) and is not illustrated.

The outer core of Pacinian corpuscles is also continuous with the perineurium (ShanthaveERAppa and Bourne, 1963) and consists of successive layers of squamous 


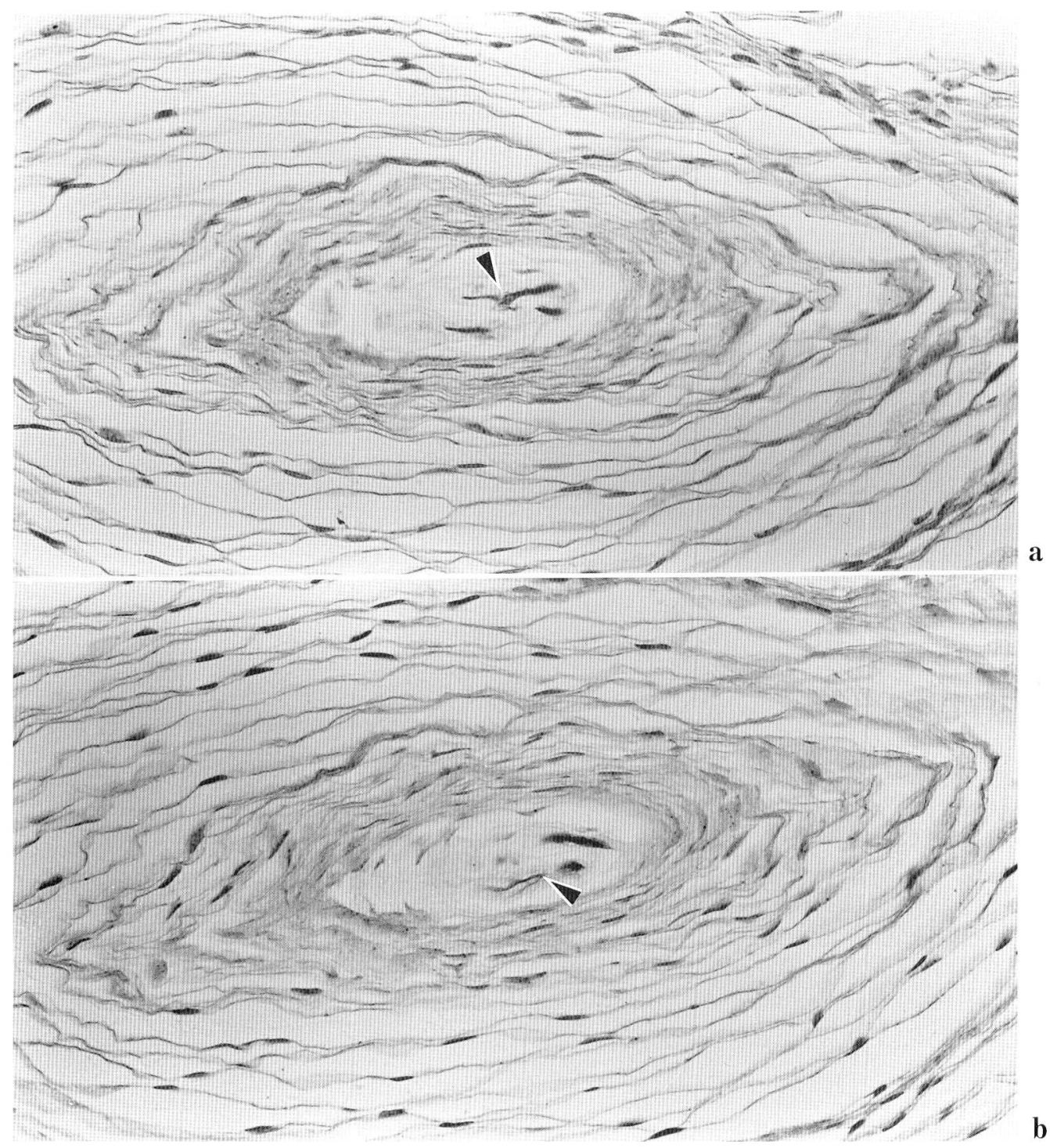

Fig. 3. $\mathbf{a}$ and $\mathbf{b}$. These photomicrographs have been taken from two sequential serial sections of a single Pacinian corpuscle of an autopsied specimen (CM) and illustrate two branch points of the central axon (arrowheads). The branches in a are coarse while the axon in $\mathbf{b}$ appears to terminate in many fine filaments. The irregular silver positivity of the axon can be correlated with the irregular distribution of neurofilaments and microtubules as noted in the following electron micrographs. a and $\mathrm{b}: \times 400$

epithelial cells (Fig. 7-9). The outer core lamellar cells have a prominent basal lamina on each surface and the cells tightly abut one another with frequent membrane densities that in the cat have been found to be tight junctions (IDE and HAYASHI, 1987). The connective tissue compartment separating successive layers of outer lamellar cells contains filamentous material of presumptive connective tissue matrix and typical 

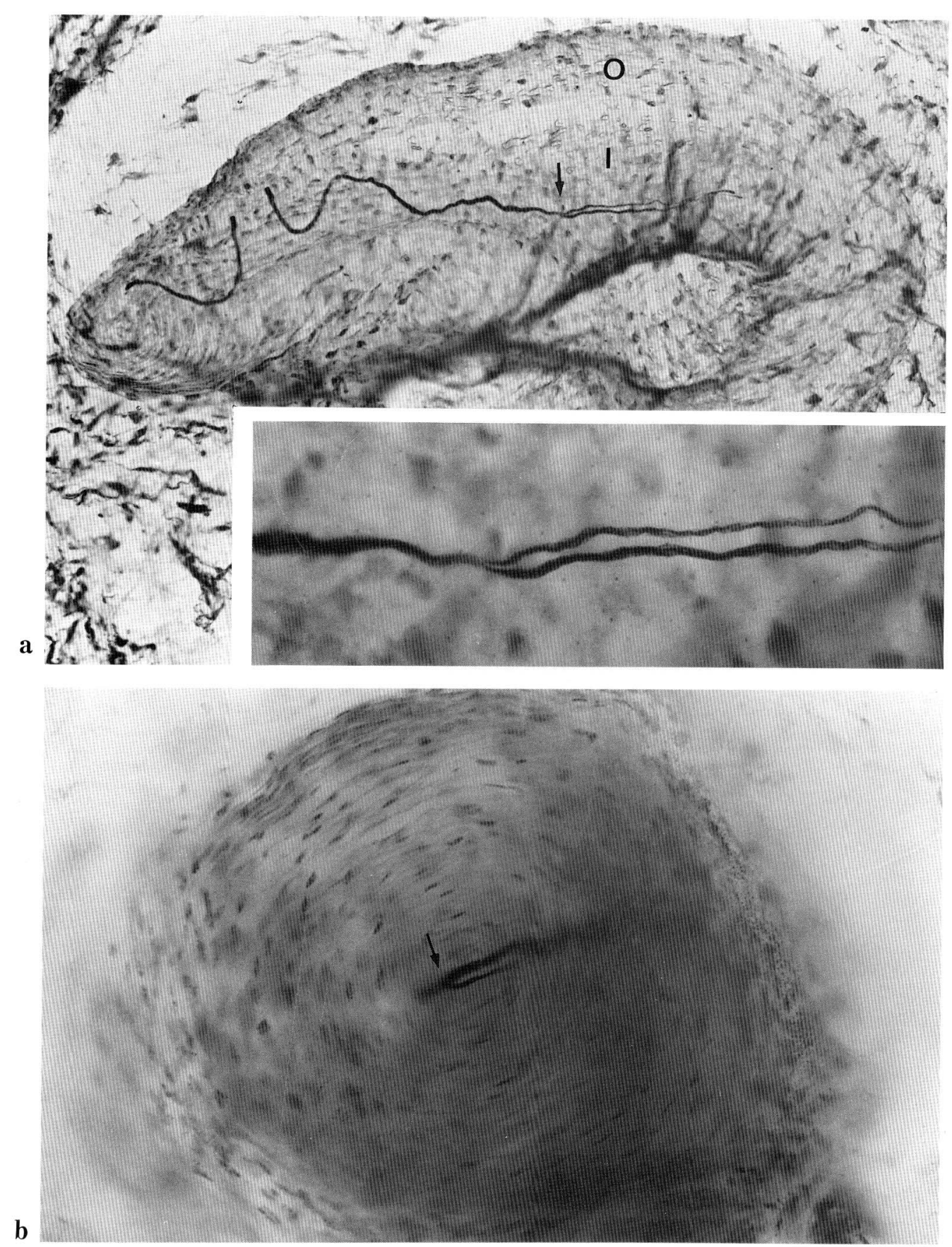

Fig. 4. a. The Pacinian corpuscle in the 28-year-old male subject. The photomicrograph was taken from $100 \mu \mathrm{m}$ thick frozen section stained with the Winkelmann silver method. The central axon (arrow) bifurcates within the inner core $(I)$ that cannot be resolved in this thick section. The outer core $(O)$ of the corpuscle is stained more dark than the surrounding connective tissue. $\times 125$. Inset. The higher magnification photomicrograph depicts the branch point as well as subtle differences in silver positivity along the course of the axon. $\times 600$. b. The Pacinian corpuscle in the thumb of the 2-year-old subject. The axon (arrow) is oriented perpendicular to the plane as depicted in Figure $4 \mathrm{a}$. The bifurcation is seen as two axons are separated by clear space. $\times 300$ 


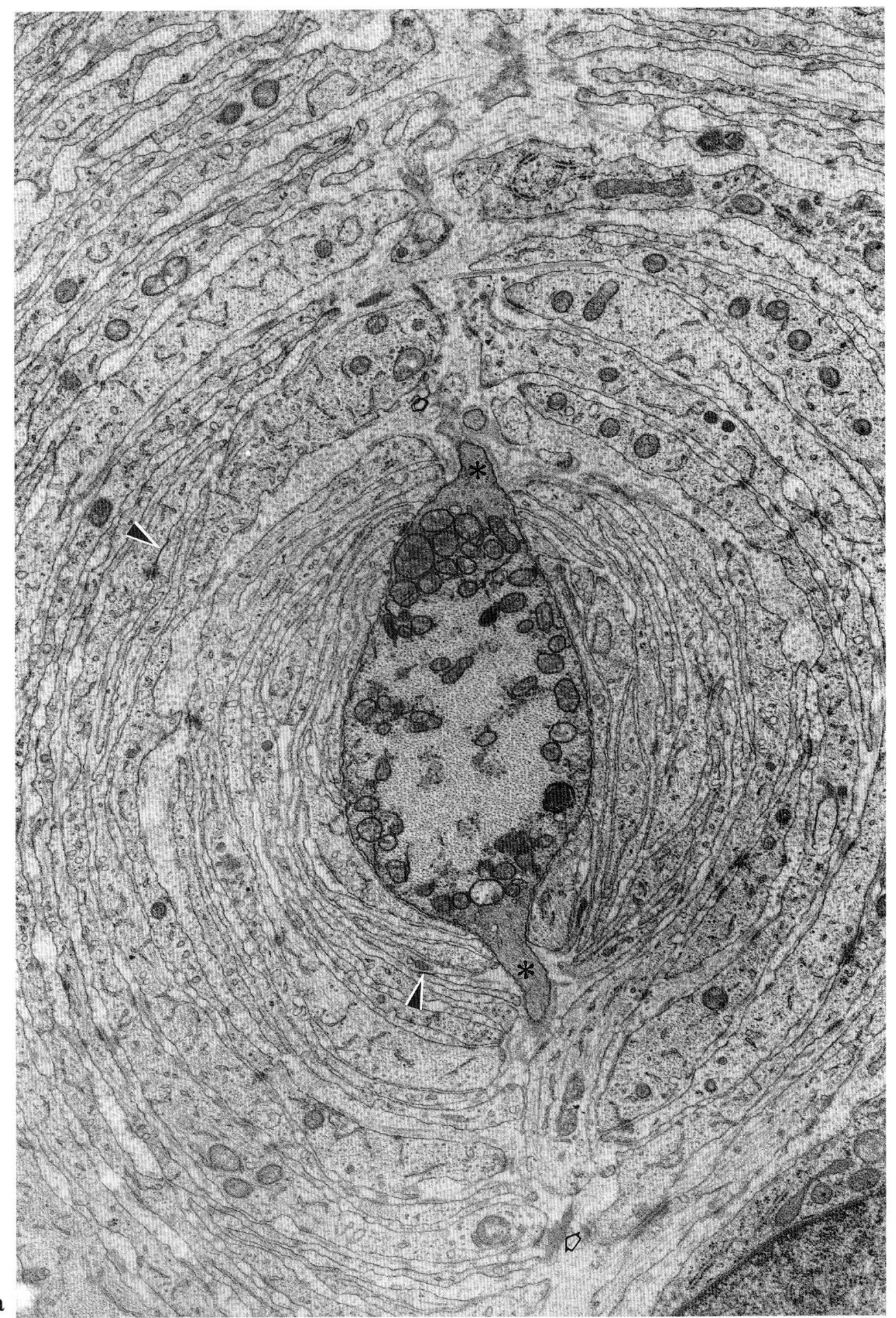

Fig. 5a. Legend on page 371. 


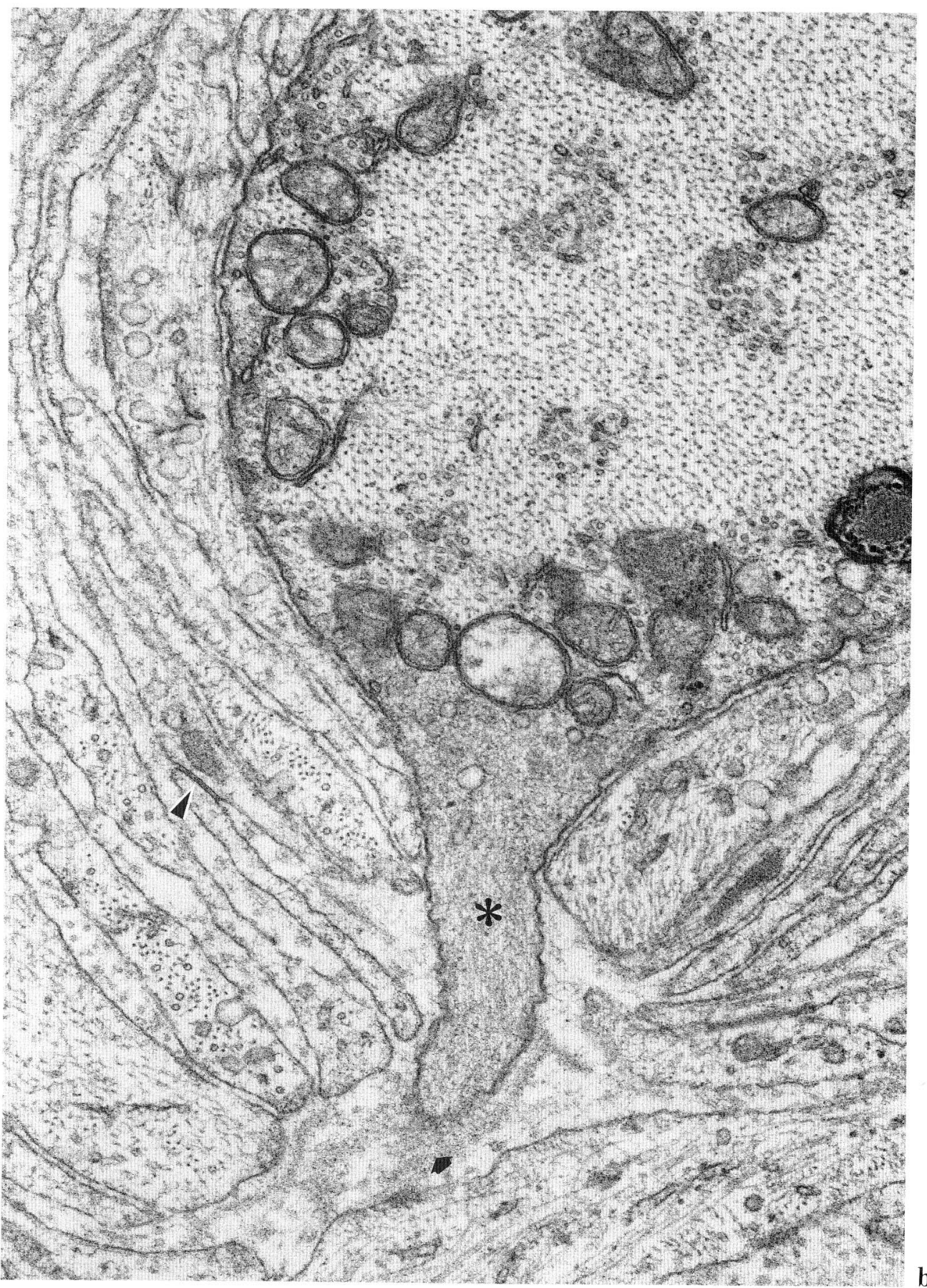

Fig. 5b. Legend on the opposite page 
collagen fibrils of approximately $250-300 \AA$ in diameter. The outer lamellae themselves contain extremely small mitochondria, numerous caveolae and scattered filaments.

The outer lamellae are separated from the inner lamellae by a layer of cells that has been called the intermediate cell layer (Fig. 7-9) by IDE and HAYASHI (1987). This layer of cells is usually double due to branching of the cell processes and the cytoplasm is characteristically electron opaque. The cells of the intermediate cell layer lack a complete basal lamina. These cells envelope the bulbous terminal ends of inner core lamellae at the inner core ends (IDE et al., 1987).

The inner core of human Pacinian corpuscles consist of the central axon terminals and associated lamellar processes of the inner core lamellae (Fig. 5). The axons usually are oval or elliptical in profile, but occasionally appear to be round in profile, and the long axis is the $\mathrm{Y}$-axis abutting the cleft in the inner core (Fig. 5). Even when circular in profile two axonal axes can usually be defined, and in some cases three distinct polar or Y-axis specializations can be identified. Axonal spines are confined to the $\mathrm{Y}$-axis in corpuscles with only a single central axon (MUNGER et al., 1987) and we will so designate the specialized regions of the central axons in human Pacinian corpuscles even though a given axon can have more than two such regions as noted below.

Typical oval axonal profiles can be characterized as housing mutually perpendicular X-and Y-axis. The X-axis is characterized by the presence of inner core lamellae directly abutting the axolemma without any intervening connective tissue matrix or collagen fibrils. The axolemma and the innermost membrane of the inner lamellae frequently have a significantly narrowed space between the two membranes. We have been unable to identify these areas as typical junctional complexes despite careful searching, and can merely note that the membranes appear to approach one another in a consistent manner suggesting a close approximation of the outer leaflets of the unit membranes.

The axons typically contain numerous mitochondria, membranous debris and lipoidal material of variable appearance. The mitochondria and other organelles tend to be clumped leaving areas of axoplasm with only neurofilaments and microtubules as suggested in silver-stained material. The axoplasm at the $\mathrm{Y}$-axis beneath the axonal spines contain a feltwork of filaments continuous with those of the axonal spines. At this site the axolemma typically contains scattered vesicles as well.

Fig. 5. $\mathbf{a}$ and $\mathbf{b}$. The inner core of a typical Pacinian corpuscle is illustrated at low and high magnification in these two electron micrographs from the same corpuscle. The lower half of the central axon of $\mathbf{a}$ is illustrated at higher magnification in $\mathbf{b}$. The central axon is elongated from the top to the bottom of the micrograph and axonal spines (*) project into the cleft of the inner core. The elongated axis of the axon is the $\mathrm{Y}$-axis. The $\mathrm{X}$-axis of the axon is to the right and left and abuts thin cytoplasmic processes of inner core lamellae. The inner core lamellae are separated by thin compartments of connective tissue that contain thin fibrils of collagen (see also Fig. $7 \mathrm{~b}$ and 8 ). The clefts in the inner core contain larger fibrils of collagen and small fibrils of elastica (empty arrow). Two gap junctions are indicated by the arrowheads and the bottom arrowhead is also included in $\mathbf{b}$. Many other membrane densities within the inner core lamellae are not identified. b illustrates an axonal spine and associated tissue of the cleft at higher magnification. The arrowhead is the same as the lower arrowhead in a and identifies a gap junction. The axonal spines $(*)$ contain numerous filaments and scattered vesicles, and partly abut basal lamina-like material (solid arrow). a : $\times 16,000, \mathrm{~b}: \times 56,000$ 


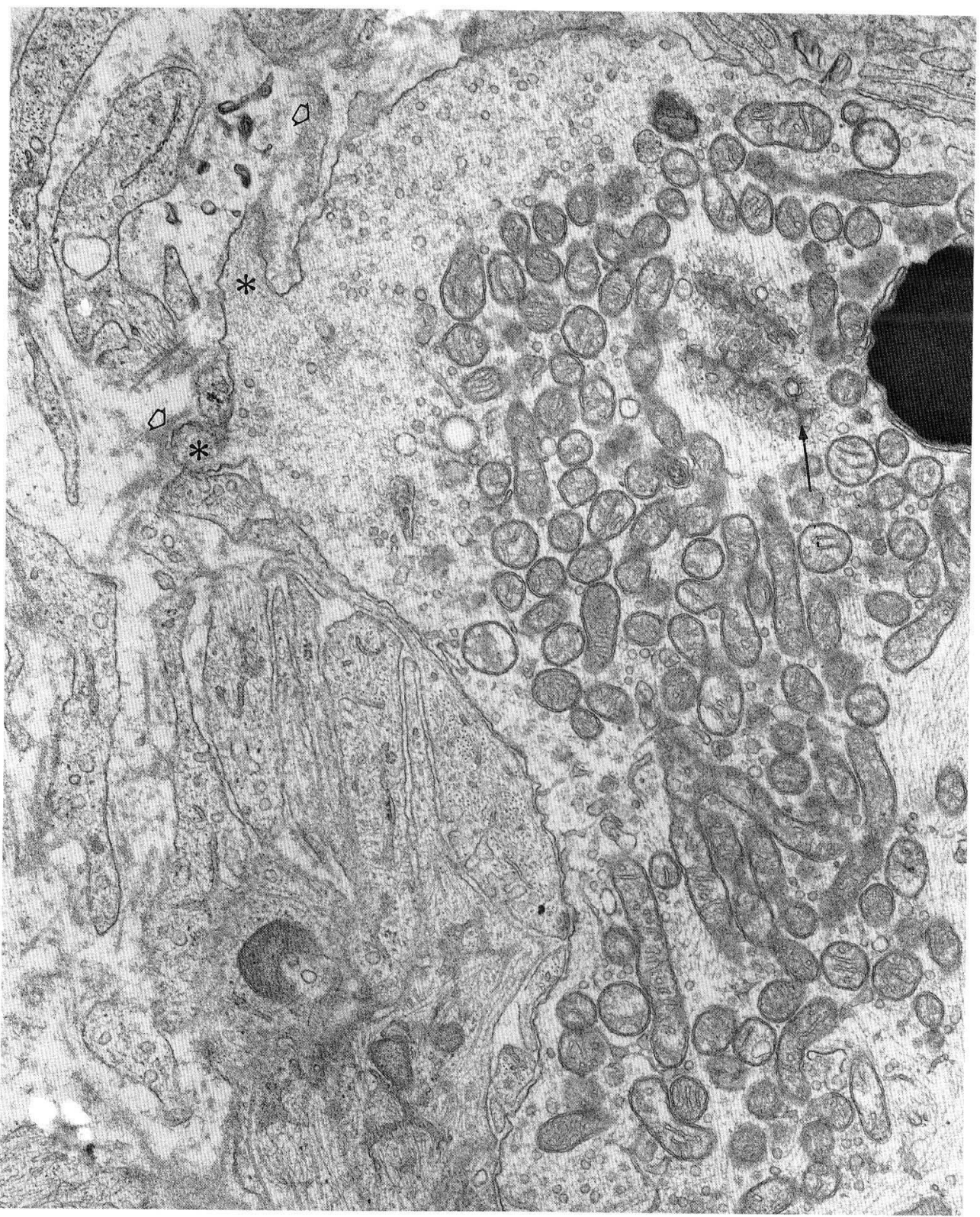

Fig. 6. a and b. The two electron micrographs illustrate portions of the extreme tip of the axon based on the identification of the extreme tip in cat Pacinian corpuscles (IDE et al., 1987). Portions of several axonal spines $(*)$ can be seen and the expanse of axoplasm surrounding them is also part of the extreme tip. The axoplasm contains masses of filaments and vesicles resembling the content of axonal spines. The spines project into an area containing masses of basal lamina-like material (empty arrows). The area of the axon to the upper half lacks the inner core lamellae that are present in the bottom half of the micrograph. The axon contains an unusual density (arrow) identical to an unidentified inclusion in a human axon terminal noted by HALATA and MUNGER (1985). In b the expanse of the axon to the left is all part of the extreme tip and only in upper right can inner core lamellae be seen. The axonal spines (*) extend into a compartment containing basal lamina-like material (empty arrow). The axolemma to the left has numerous membrane densities and contains numerous filaments and vesicles that identify this area as part of the extreme tip. a: $\times 21,000$, b: $\times 26,000$ 


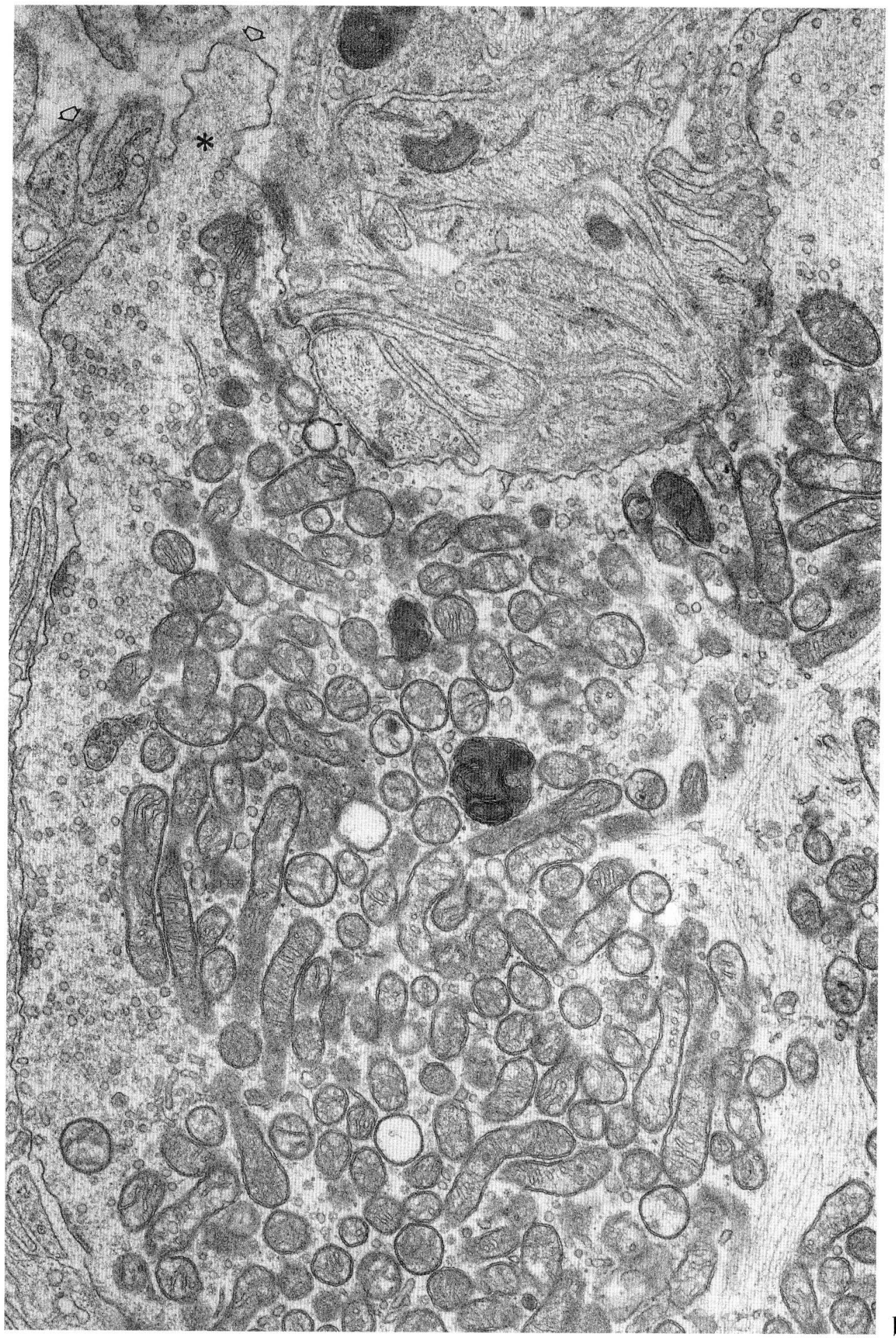




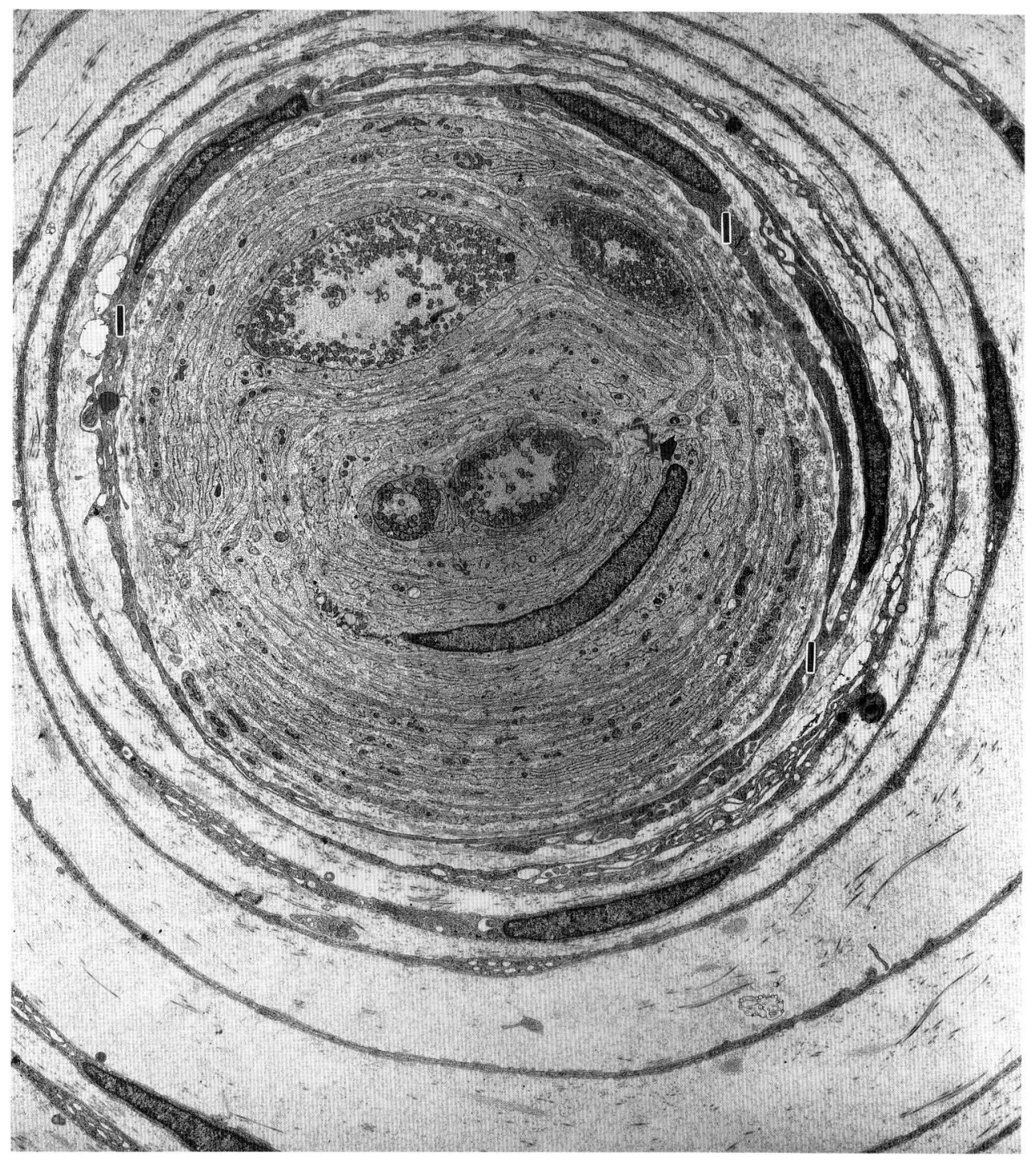

Fig. 7. a. and b. A Pacinian corpuscle with multiple axons in the inner core is illustrated at a survery magnification (a) and at a higher magnification (b). The outer core is also illustrated in a. The inner core contains two central axonal profiles that are illustrated at a higher magnification in $\mathbf{b}$. The layer of cells between the inner and outer cores is identified as the intermediate layer $(I)$ and lacks a continuous basal lamina as noted at higher magnification in Figures 8 and 9. The black arrow depicts an fibril of elastica in both $\mathbf{a}$ and $\mathbf{b}$. The cleft of the inner core extends from left to right and is indicated by the two central axonal profiles. The upper two axonal profiles are within the same inner core half. This low magnification micrograph thus depicts the fact that only one inner core is present even though the upper two axonal profiles would appear to create another inner core. In $\mathbf{b}$ the cleft is indicated by the elastic fibril (arrow). Axonal spines $(*)$ project into the cleft and also towards the inner core lamellae. The extremely small collagen fibrils of the inner core connective tissue compartments are stained with good contrast in this micrograph. a: $\times 4,800$, b: $\times 12,200$ 


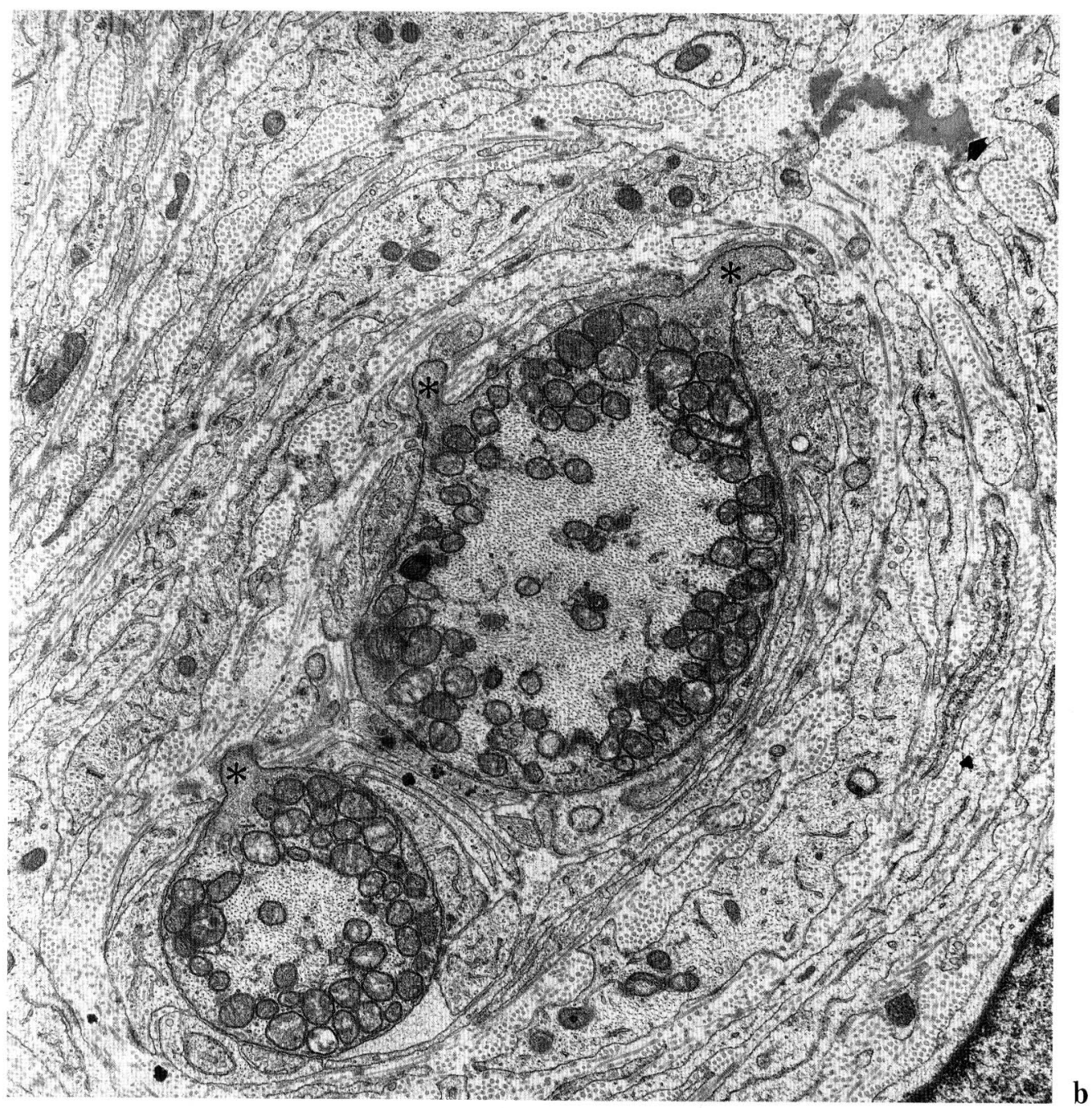

Axonal spines of the central axons project into the clefts in the inner core. Axonal spines are variable in appearance with the profile of some short and stubby and others long and cylindrical. In most cases they are filled with tightly packed bundles of filaments the nature of which has not been determined to date. The axonal spines are enveloped by numerous fine fibrils of the connective tissue that are continuous with the fibrils of the clefts.

The inner lamellae themselves are thin sheets of cytoplasm stacked on top of the other with an intervening layer of connective tissue matrix. The lamellae contain numerous vesicles, small mitochondria and filaments. The plasma membranes of inner lamellae frequently have junctional complexes consistent in appearance with gap junctions (Fig. 5). The latter have been identified in cat Pacinian corpuscles in a freezefracture preparation and those dipicted in Figure 5 are consistent with those noted in the cat (IDE and HAYASHI, 1987). Gap junctions are identified in human Pacinian corpuscles as areas of tight apposition of adjacent plasma membranes that are straight 


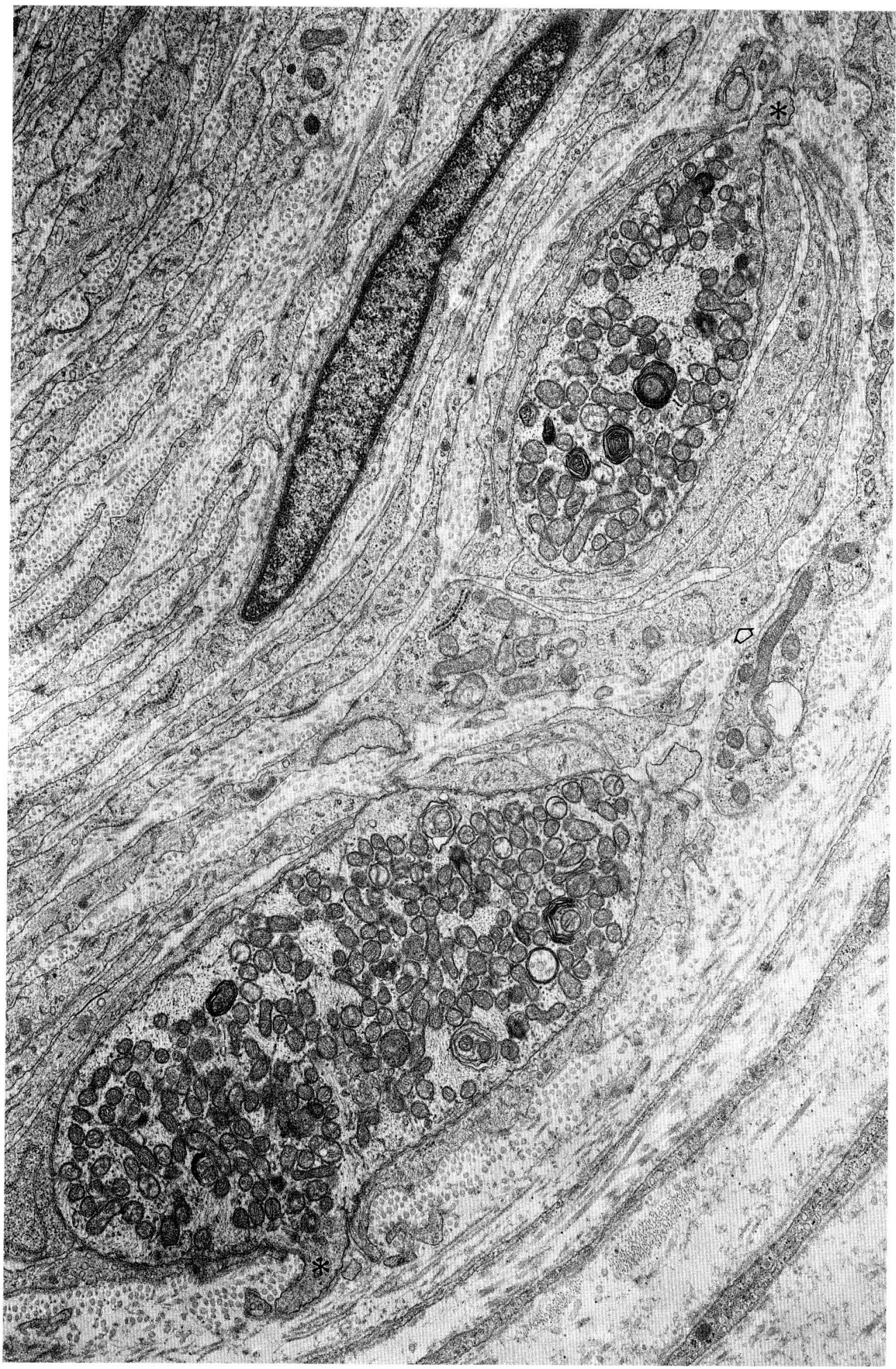

Fig. 8. Legend on the opposite page. 
and parallel, and the intermembranous space contains a faint density. The identification is presumptive pending confirmatory freeze-fracture preparations. In other cases, the membranes of abutting inner lamellae, or even the inner lamellae and the axolemma, have membrane densities identified as intermediate junctions and they thus resemble fascia adherens of other epithelia.

The ultraterminal region of human Pacinian corpuscles resembles the equivalent region of cat Pacinian corpuscles as described by SPENCER and SCHAUMBURG (1973) and IDE et al. (1987). The inner lamellae become sparse, and no longer piled one on top of the other. In Figure 6 we have approached the extreme tip as we begin to encounter numerous axonal spines and a feltwork of filaments in the subaxolemmal axoplasm. This particular example has a profusion of vesicles resembling synaptic vesicles as well as membranous densities underlying the axolemma. Such densities and accumulations of vesicles have not been observed with regularity in cat Pacinian corpuscles, but several examples are present in Figure $6 \mathrm{~b}$.

The connective tissue compartment between the lamellae contain numerous small collagen fibrils the diameter of which often measures approximately 150-200. . This diameter is consistent with that observed in the cat (MUNGER et al., 1987) but is significantly less than typical endoneurial collagen fibrils as noted by OSAWA and IDE (1986). The collagen fibrils between the lamellae blend with the connective tissue compartment of the clefts that contain larger collagen fibrils and fibrils of elastica (Fig. la, b).

\section{Electron microscopy-multiple axons}

Multiple axons within the inner core have been traced in sequential step semithin and thin sections through a single Pacinian corpuscle and observed in random sections in other corpuscles. When multiple axons are present, a central axon or pair of axons is clearly present as illustrated in Figure 7. The central axons always interface the cleft of the inner core. Thus axonal spines project from the Y-axis into the cleft in most cases. In other cases the branches of the central axon fail to have typical $\mathrm{Y}$-axis axonal spines and the axonal spines project away from the $\mathrm{Y}$-axis. Thus one striking difference in corpuscles with multiple axons is the lack of uniformity of the location of axonal spines which normally projected only towards the cleft as in Figure 1. The other faces of the central axon still tightly abut lamellae of inner core cells and thus are the $\mathrm{X}$-axes of the central axons.

The other axons present in the inner core typically are present at the junction of the inner and outer cores and thus often associated with cells that would normally be identified as the intermediate cell layer noted in the cat by the present authors (MUNGER et al., 1987; IDE et al., 1987). The intermediate cell layer in the cat is typically composed of one or more cell layers due to branching of the cells and Figure 7 has typical intermediate cell layers present. The multiple axons of the corpuscles in Figure 7 are thus within the first layers of the inner core. The more typical appearance of multiple axons is depicted in Figures 8 and 9 described below. The intermediate cell

Fig. 8. Profiles of two axons are illustrated at the junction between the inner core (upper left) and outer core (lower right). The axons are associated with cells of outermost lamellae of the inner core with the incomplete investment of basal lamina (clear arrow). The axons have axonal spines $(*)$ that project towards the connective tissue. The cells of the outer lamellae (lower right) have distinct basal laminae. $\times 21,000$ 




Fig. 9. An axon at the junction of inner and outer cores is illustrated at higher magnification similar to those seen in Figure 8. An axonal spines $(*)$ projects towards a cell of the inner lamellae. Clear arrows show patchy or irregular basal laminae on peripheral lamellae of the inner core. The extremely small diameter of collagen fibrils in the connective tissue compartment separating cells of the inner lamellae can be seen in the upper right. The lower left represents the outer core where the cells have typical basal lamina and the diameter of collagen fibrils is more typical of other tissues. $\times 24,000$ 


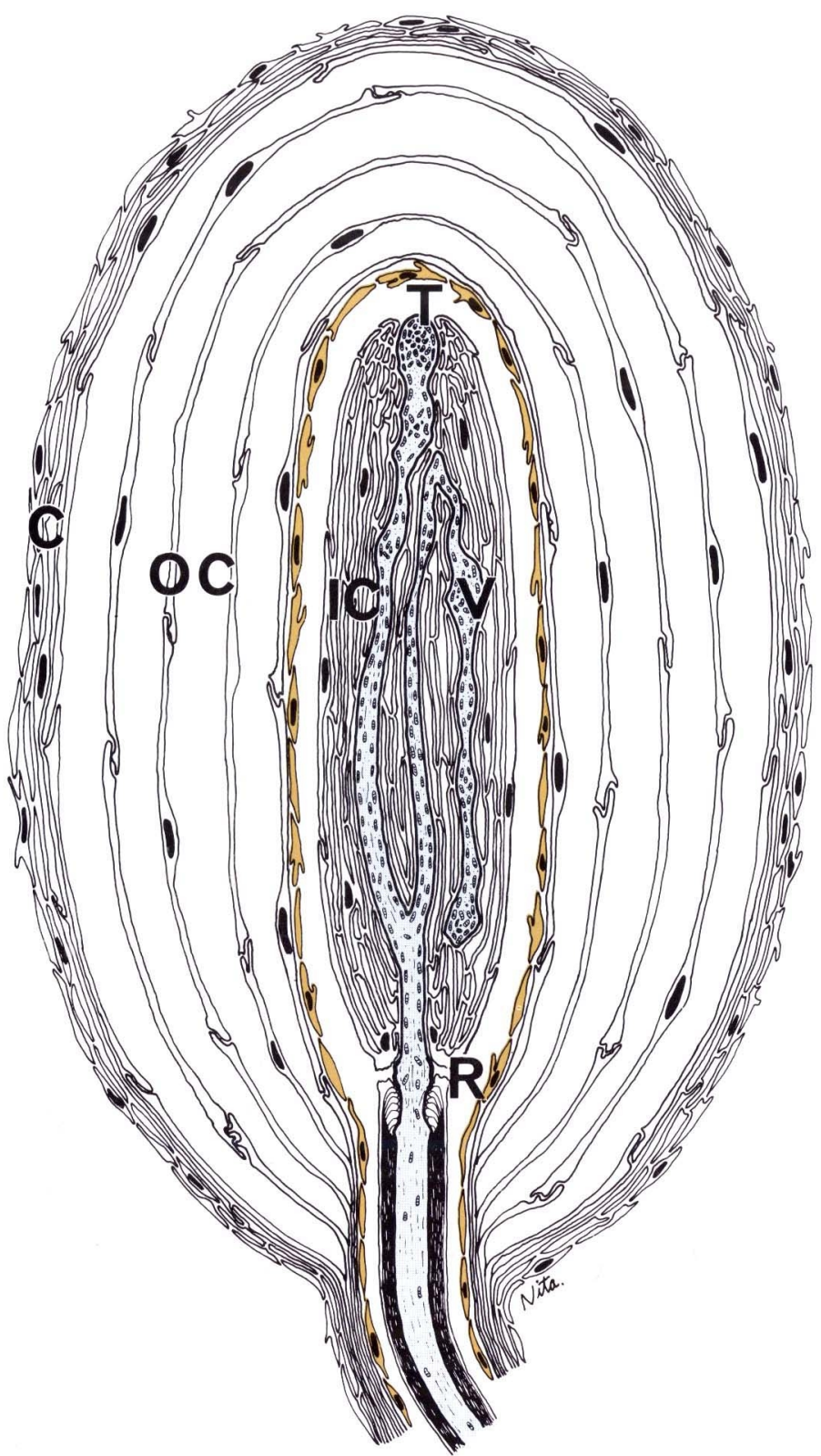

Fig. 10. This schematic drawing of a Pacinian corpuscle depicts the pattern of branching of the central axon. This illustration was drawn on a basis of observations by step thin sections of a Pacinian corpuscle obtained from 20-year-old volunteer. The axon (colored in blue) is divided into two branches at the proximal portion of the inner core, one of which extends all the way to the end of the inner core terminating therein as an extreme tip ( $T$ ) of the axon, while the other, after coursing for a short length along the companion to the distal direction, turns back to the proximal direction and ends near the branching point. This latter branch is partly enlarged as varicosities $(V)$ which present the structure similar to the extreme tip of the central axon. In addtion, part of this branch abuts during its course the intermediate layer cells (colored in brown). Such a contact between axons and intermediate layer cells is not illustrated in this drawing. $C$ capsule, $I C$ inner core, $O C$ outer core, $R$ last node of Ranvier. 
layer in Figure 7 thus resembles that of the normal cat corpuscle and is typical for human corpuscles with only one central axon.

The multiple axons associated with the intermediate cell layer and thus present at the junction of the inner and outer core are variable in appearance. Those depicted in Figures 8 and 9 are typical and are large expanses of axoplasm confirmed on serial section to be cross sections. The diameter would conform to the diameter of the axons noted by light microscopy in Figures 3 and 4. The axons have axonal spines that project apparently randomly into the adjacent lamellae of the inner core or into the space between intermediate layer cells. The cells of the intermediate layer do not have a continuous basal lamina in the cat (see above) and in these cases the intermediate cell layers are characterized by irregular patches of basal lamina that are not the same as the outer lamellae as noted in both Figures 8 and 9 . We have not observed cell junctions to be present consistently in these apparantly modified intermediate layer cells.

When multiple axons are present in the inner core, only one inner core with bilateral symmetry of each half can be found. This important point will be addressed in the Discussion as the basis for determining the absence of neural regeneration in the present cases. In Figure 7 an axis of symmetry of the inner core is present that is identical to the symmetry of Figure 1 . The cleft clearly divides the halves of the inner core into bilaterally symmetrical components and the multiple axons do not have separate inner cores.

A schematic drawing of branching of the central axon is illustrated in Figure 10, which was drawn on the basis of observations by sectioning of a Pacinian corpuscle obtained from a 20-year-old volunteer.

\section{DISCUSSION}

Human Pacinian corpuscles as observed in the present study resemble their counterparts described to date in the cat, but evidence a remarkable diversity of axonal profiles in the inner core that are only consistent with the hypothesis that sprouting of sensory axons is normal in the inner core of human corpuscles. The individual authors themselves were skeptical of their own data and concluded prior to the present study that each possessed abnormal material. Yet we have recently had an opportunity to review human digital skin following nerve regeneration and can conclude that the material that we describe in the present report is indeed normal as the changes in reinnervated Pacinian corpuscles are pathognomonic for regeneration (MUNGER and IDE, unpublished observations; ZELENÁ, 1984a, b)

Apart from the inner core, Pacinian corpuscles in man resemble those present in the cat as described previously (PEASE and Quilliam, 1957; POLAĆĚK and MAZANEC, 1966; Nishi et al. 1969; Chouchkov, 1971; Munger, 1971; Spencer and Schaumburg, 1973; Halata, 1975; IdE and Saito, 1980; MalinovsKÝ and PÁč, 1982 ; MunGer et al., 1987; IDE et al., 1987; IDE and HAYASHI, 1987). The capsule and outer core of the corpuscle are both continuous with the perineurium of the innervating nerve fiber as described originally by SHANTHAVEERAPPA and BOURNE (1963). Each layer of the outer core and each layer of the capsule are a complete epithelium composed of squamous cells with specialized intercellular junctional complexes and ensheathed on both sides with basal lamina. The presence of numerous tight junctions especially in the innermost layers of the outer lamellae as described by IDE and HAYASHI (1987) strengthen the concept that these cellular layers are in fact an epithelium, and each is a completely 
separate epithelium system. The connective tissue between each successive lamellae is the stroma for two separate lamellae and a basal lamina is present at the epithelial/ connective tissue junction.

However, tight junctions of the inner portion of the outer lamellae would also electrically isolate the inner core if these tight junctions are indeed extensive enough to accomplish an electrotonic isolation. Based on the lengths of tight junctions noted by IDE and HAYASHI (1987), we would conclude that until microelectrode penetrations can clarify the ionic environments of the inner and outer core we must assume that the tight junctions would electrotonically isolate the inner core.

The general organization of the lamellae of the inner core again resembles that described in the cat. The individual lamellae are extremely thin cytoplasmic processes of specialized cells and they lack a basal lamina, but do abut an unusual connective tissue compartment. The connective tissue contains unusual collagen fibrils, not even resembling those of the endoneurium of typical peripheral nerves as describe by OSAWA and IDE (1986). The inner core has been noted in the cat to have some features in common with other epithelia and the presence of an unusual collagen fibril is one more example.

The presence of junctional complexes between the lamellae including presumptive gap junctional complexes between the lamellae confirms the observation of IDE and HAYASHI (1987) in the cat using freeze-fracture techniques. Gap junctions are easy to identify near the clefts of each half of the inner core. This type of structural coupling of cells would imply electrotonic coupling of the halves of the inner core as well. The ionic environment of the axon is thus markedly different on its $\mathrm{X}$ - and $\mathrm{Y}$-axis, especially considering the presence of axonal spines only abutting the cleft at the $\mathrm{Y}$-axis. The inner core lamellae would isolate the $\mathrm{X}$-axis faces of the axon and thus restrict the flow of current of ions to the $\mathrm{Y}$-axis as described in more detail by MUNGER and IDE (1987).

However, the most intriguing feature of human Pacinian corpuscles is the redundant axonal profiles that on reconstruction turn out to be branches of a single innervating axon. Furthermore they in no way resemble the axons that are typically multiple and/or branches of the same axon in reinnervated Pacinian corpuscles as described by ZELENÁ (1984a, b). The fact that we have also identical finding in human reinnervated corpuscles (MUNGER and IDE, unpublished observation) means that the present material had not been denervated. Each of these points will be discussed briefly to clarify the important relationships of the inner core.

First, the axons clearly branch in the inner core. The continuity of axons and fine branches has been repeatedly seen and traced in serial paraffin sections. Thus we are forced to recognize that these fine axonal branches are sprouts from a single parent axon. We do not see signs of multiple innervation of human Pacinian corpuscles despite reports to the contrary (SANTINI, 1969). If unmyelinated or autonomic fibers are common in human or cat Pacinian corpuscles, our material must be very exceptional. Otherwise we are forced to conclude that Pacinian corpuscles do not have a prominent autonomic innervation. The presence of A-delta fibers in Meissner corpuscles has however been conclusively demonstrated in the monkey lip by HALATA and MUNGER (1983). In that site free nerve endings are numerous in association with Meissner corpuscles.

We have been able to confirm ZELENÁ (1984b) in a recent study of human digital skin following reinnervation (MUNGER and IDE, unpublished observations). As noted by ZELENÁ (1984a) the inner core of Pacinian corpuscles rapidly degenerates and atrophies 
following denervation. Upon reinnervation several branches of axons are typically observed and each is surrounded only by a thin layer of inner core-like material. Our light microscopic examples of reinnervated human Pacinian corpuscle are identical to ZELENÁ's, and we can see individual axons surrounded by a thin layer of inner core. The present results are thus proof that the present material is normal in that a healthy inner core lamellae are present and many stacks of lamellae envelope the $\mathrm{X}$-axis of axons.

The findings of regeneration of cryo-treated Pacinian corpuscles are remarkable in that the extracellular matrix of the inner core can serve as the site of development of a new inner core by regenerating axons and Schwann cells (IDE, 1987). By cryotreatment the cellular components of Pacinian corpuscles were damaged, leaving only the frameworks of the extracellular matrix of the corpuscles in situ. Regenerating axons extend from the proximal intact portions of the nerve and enter such acellular frameworks of the old corpuscles. Such regenerating axons usually gave several branches within the old inner core zone, each of which was surrounded by lamellar cells differentiated from immature Schwann cells. This phenomenon indicates that the inner core of the Pacinian corpuscle offers a unique micro-environment in which axons can easily sprout, giving several branches in the adult normal corpuslces.

The above leads us to conclude that the inner core of Pacinian corpuscles is most unique from the point of view of trophic interactions that occur when nerves normally grow into these corpuscles, and also when nerves re-grow into these corpuscles. Pacinian corpuscles in man can be typically reinnervated, the same can not be said for other receptors in the skin. In fact only a minority of Meissner corpuscles will be normally reinnervated in cases where every Pacinian corpuscle is reinnervated. Human Pacinian corpuscles thus contain substances promoting axonal sprouting in the normal as well as denervated tissue.

Acknowledgements. The authors wish to acknowledge the valuable assistance of the volunteers (especially, Mr. Kuniya Koizumi, medical student of Iwate Medical University). We also thank Dr. S. IZAKI, Department of Dermatology, for surgical biopsy of the material, and Miss E. YoshidA for typing the manuscript. Material was also donated for research $(\mathrm{CM})$ through the provisions of the Pennsylvania (U.S. A.) Uniform Anatomical Gift Act and the gifts are gratefully acknowledged. The study was supported in part by grant from the US Public Health Service NS 19462. The authors wish to express a special debt of gratitude to the Japan Society for the Promotion of Sciences who supported a Fellowship for BLM that made this joint effort possible.

\section{REFERENCES}

Chouchkov, H. N.: Ultrastructure of Pacinian corpuscles in men and cats. Z. mikrosk.-anat. Forsch. 83: 17-32 (1971).

Halata, Z.: The mechanoreceptors of the mammalian skin. Ultrastructure and morphological classification. Adv. Anat. Embryol. Cell Biol. 50: 7-75 (1975).

Halata, Z. and B. L. Munger: The sensory innervation of primate facial skin. II: Vermilion border and mucosa of lip. Brain Res. Rev. 5: 81-107 (1983).

Ide, C.: Role of extracellular matrix in the regeneration of a Pacinian corpuscle. Brain Res. 413: 155-169 (1987) 
Ide, C. and T. Saito: Electron microscopic histochemistry of cholinesterase activity of Vater-Pacini corpuscle. Acta histochem. cytochem. 13: 298-305 (1980).

Ide, C. and S. Hayashi: Specialization of plasma membranes in Pacinian corpuscles: Implications of mechano-electric transduction. J. Neurocytol. (1987, in press)

Ide, C., Y. Yoshida, S. Hayashi, M. Takashio and B. L. Munger: A re-evaluation of the cytology of cat Pacinian corpuscles, II. The extreme tip. Cell Tiss. Res. (1987, in press).

Malinovský, L. and L. Páč: Morphology of sensory corpuscles in mammals. J. E. Purkyně University Brno, Medical Faculty, Brno, 1982.

Munger, B. L. : Patterns of organization of peripheral sensory receptors. In: (ed. by) W. R. Loewenstein: Handbook of sensory physiology, Vol. 1. Springer, New York, 1971 (p. 523-556).

Munger, B. L. and C. Ide: The enigma of sensitivity in Pacinian corpuscles: A critical review and hypothesis of mechano-electric transduction. Neurosci. Res. (1987, in press).

Munger, B. L., Y. Yoshida, S. Hayashi, T. Osawa and C. Ide: A re-evaluation of the cytology of Pacinian corpuscles. I. The inner core and clefts. Cell Tiss. Res. (1987, in press).

Nishi, K., C. Oura and W. Pallie: Fine structure of Pacinian corpuscles in the mesentery of the cat. J. Cell Biol. 43: 539-553 (1969).

Osawa, T. and C. Ide: Changes in thickness of collagen fibrils in the endo- and epineurium of the mouse sciatic nerve during development. Acta anat. 125: 245-251 (1986).

Pease, D. C. and T. A. Quilliam: Electron microscopy of the Pacinian corpuscle. J. biophy. biochem. Cytol. 3: 331-342 (1957).

Poláček, P. and K. Mazanec: Ultrastructure of mature Pacinian corpuscles from mesentery of adult cat. Z. mikrosk-anat. Forsch. 75: 343-354 (1966).

F. Rice and B. L. Munger: A comparative light microscopic analysis of the sensory innervation of the mystacial pad. II. The common fur between the vibrissae. J. comp. Neurol. 252: 186-205 (1986).

Santini, M.: New fibers of sympathetic nature in the inner core region of Pacinian corpuscles. Brain Res. 16: 535-538 (1969).

Sevier, A. C. and B. L. Munger: A silver method applicable to paraffin sections of formolfixed tissue. J. Neuropathol. exp. Neurol. 24: 130-135 (1965).

Shanthaveerappa, T. R. and G. H. Bourne: New observations on the structure of the Pacinian corpuscle and its relation to the perineural epithelium of peripheral nerves. Amer. J. Anat. 112: 97-109 (1963).

Spencer, P. S. and H. H. Schaumburg: An ultrastructural study of the inner core of the Pacinian corpuscle. J. Neurocytol. 2: 217-235 (1973).

Winkelmann, R. K.: Nerve endings in normal and pathologic skin. Thomas, Springfield, Illinois, 1960.

Zelená, J.: The effect of long-term denervation on the ultrastructure of Pacinian corpuscles in the cat. Cell Tiss. Res. 238: 387-394 (1984a). : Multiple axon terminals in reinnervated Pacinian corpuscles of adult rat. J. Neurocytol. 13: 665-684 (1984b).

井出 千束

厂 020 盛岡市内丸 $19-1$

岩手医科大学

解剖学第二講座
Prof. Chizuka IDE

Department of Anatomy

School of Medicine

Iwate Medical University

Morioka, 020 Japan 